\title{
Analytical and numerical treatment to the (2+1)-dimensional Date-Jimbo-Kashiwara-Miwa equation
}

https://doi.org/10.1515/nleng-2021-0014

Received Mar 8, 2021; accepted Jun 9, 2021.

\begin{abstract}
In this work, we study the $(2+1)$-dimensional Date-Jimbo-Kashiwara-Miwa (DJKM) equation. We employ the extended tanh function method and the simple equation method to achieve analytical soliton solutions. Moreover, numerical treatment for this equation is introduced by the finite difference method. We justify the accuracy of the obtained results by exhibiting illustrative tables and proper graphs.
\end{abstract}

Keywords: Date-Jimbo-Kashiwara-Miwa equation; The simple equation method; The extended tanh function method; Soliton solution; Finite difference method

\section{Introduction}

The Kadomtsev-Petviashvili (KP) equation is one of the basic soliton equations and it has fundamental significance in mathematical physics. The Date-JimboKashiwara-Miwa (DJKM) equation is a member of the KP hierarchy equations that can be takes the form $[1,2]$

$u_{x x x x y}+4 u_{x x y} u_{x}+2 u_{x x x} u_{y}+6 u_{x y} u_{x x}-\alpha u_{y y y}-2 \beta u_{x x t}=0$,

where $u(x, y, t)$ is the wave amplitude function and $t$ is time. The DJKM equation describes the long water waves with frequency dispersion and weakly nonlinear restoring forces. This equation has been investigated by employing a variety of poweful methods such as, the Hirota bilinear method [1], the improved $\tan (\phi(\xi) / 2)$-expansion method [3], the extended transformed rational function

*Corresponding Author: Khalid K. Ali, Mathematics Department, Faculty of Science, Al-Azhar University, Nasr-City, Cairo, Egypt, E-mail: khalidkaram2012@azhar.edu.eg

Mona S. Mehanna, Faculty of Engineering, MTI University, Cairo, Egypt, E-mail: monamehanna@gmail.com

Abdul-Majid Wazwaz, Department of Mathematics, Saint Xavier University, Chicago, IL 60655, USA, E-mail:wazwaz@sxu.edu algorithm [4], the $\exp -(\phi(\xi))$ method [5], multiple expfunction method [6], the Wronskian and Grammian techniques [7], Wazwaz [2] confirmed the Painlevé integrability of this equation, where multiple soliton solutions of (2+1)-dimensional DJKM equation [2] were formally derived. Finding analytical and numerical solutions to the equations plays an important role in understanding natural phenomena see that in [8-16].

In this work, the extended tanh function method [1719] and the simple equation method [20, 21], will be used to find analytical solutions to the above-mentioned DJKM equation. Moreover, the finite difference method will be employed to obtain numerical solution to the DJKM equation.

This paper is organized as follows: In section 2, we present outlines of the methods that will be used. The seating solutions to the DJKM equation were given in section 3. In section 4, the numerical solutions for that equation are introduced. Some graphical illustrations are introduced in section 5. Finally, the conclusion of our work is given.

\section{Outlines of the methods}

\subsection{The extended tanh function method}

Suppose the nonlinear partial differential equation as

$$
F\left(u, u_{t}, u_{x}, u_{y}, u_{x t}, u_{x x}, u_{y t}, u_{y y}, \ldots\right)=0 \text {, }
$$

where $u=u(x, y, t)$ is the unknown function, $F$ is a polynomial in $u$ and its partial derivatives.

Step 1: To convert (2) to an ODE, we use the wave transformation:

$$
u(x, y, t)=U(\xi), \quad \xi=a x+b y-c t,
$$

where $a, b$ are constants and $c$ is the velocity of the traveling wave. Consequently, (2) becomes an ordinary differential equation:

$$
H\left(U^{\prime}, U^{\prime \prime}, U^{\prime \prime \prime}, \ldots\right)=0 .
$$


Step 2: The modified extended tanh method presents the wave solution of (4) in the form of a finite series:

$$
U(\xi)=a_{0}+\sum_{i=1}^{N}\left(a_{i} \Phi(\xi)^{i}+b_{i} \Phi(\xi)^{-i}\right),
$$

where $\Phi=\Phi(\xi)$, is a solution of the Riccati equation

$$
\frac{d \Phi}{d \xi}=w+\Phi^{2}
$$

The Riccati equation (5) exhibits the general solutions

If $w<0$ then,

$$
\begin{aligned}
& \Phi(\xi)=-\sqrt{-w} \tanh (\sqrt{-w} \xi), \\
& \Phi(\xi)=-\sqrt{-w} \operatorname{coth}(\sqrt{-w} \xi) .
\end{aligned}
$$

If $w=0$ then,

$$
\Phi(\xi)=-\frac{1}{\xi}
$$

If $w>0$ then,

$$
\begin{aligned}
& \Phi(\xi)=\sqrt{w} \tan (\sqrt{w} \xi), \\
& \Phi(\xi)=-\sqrt{w} \cot (\sqrt{w} \xi) .
\end{aligned}
$$

Step 3: In (5), $N$ is a positive integer to be determined by balancing the highest order derivative term with the highest power nonlinear term in (4). Substituting (5) and (6) into (4) and then set the coefficients of $\Phi(\xi)^{i}$ equal to zero, we get a system of algebraic equations for $w, a_{0}, \ldots, a_{N}, b_{1}, \ldots, b_{N}$ and we solving this system to find all constants.

\subsection{The simple equation method}

We next introduce the steps to the method.

Step 1: Suppose the solution of (4) takes the form of a finite series

$$
U(\xi)=\sum_{i=0}^{N}\left(A_{i}(\phi(\xi))^{i},\right.
$$

where $A_{i}(i=0,1,2, \ldots, N), A_{N} \neq 0$, are constants to be calculated and $\phi(\xi)$ is a function satisfying the simple equations that called the Bernoulli and Riccati equations.

Step 2: In (10), $N$ is a positive integer to be determined by balancing the highest order derivative term with the highest power nonlinear term in (4).

\section{Step 3: The Bernoulli equation:}

The Bernoulli equation

$$
\phi^{\prime}(\xi)=B_{1} \phi^{2}(\xi)+B_{2} \phi(\xi),
$$

provides the the solution

$$
\phi(\xi)=-\frac{B_{2} \exp \left(\xi B_{2}+c_{1} B_{2}\right)}{-1+B_{1} \exp \left(\xi B_{2}+c_{1} B_{2}\right)},
$$

where $c_{1}$ is the constant of integration. In view of this, we examine two special cases for Bernoulli equation:

\section{Case one:}

For the equation

$$
\phi^{\prime}(\xi)=-\phi^{2}(\xi)+B_{2} \phi(\xi),
$$

we get the solution:

$$
\phi(\xi)=\frac{B_{2} \exp \left(\xi B_{2}\right)}{\exp \left(\xi B_{2}\right)-\exp \left(c_{2} B_{2}\right)},
$$

where $c_{2}$ is the constant of integration.

\section{Case two:}

For the equation

$$
\phi^{\prime}(\xi)=-\phi^{2}(\xi)+\phi(\xi),
$$

we get the solution:

$$
\phi(\xi)=\frac{\exp (\xi)}{\exp (\xi)+\exp \left(c_{3}\right)},
$$

where $c_{3}$ is the constant of integration.

The Riccati equation:

The Riccati equation

$$
\phi^{\prime}(\xi)=B_{1} \phi^{2}(\xi)+B_{2},
$$

yields the solution

$$
\phi(\xi)=\sqrt{\frac{B_{2}}{B_{1}}} \tan \left(\xi \sqrt{B_{1} B_{2}}+c_{4} \sqrt{B_{1} B_{2}}\right),
$$

where $c_{4}$ is the constant of integration.

Step 4: Substitute from (10) and one of above cases into (4), and equating each coefficient of the polynomial to zero, then we get a system of algebraic equations in $A_{i}, B_{1}, B_{2}, a, b, c$, which can be solved by Mathematica program. 


\section{Soltion solutions to the DJKM equation}

Applying the transformation (3) into (1), we get the following ordinary differential equation:

$$
6 a^{3} b U^{\prime \prime}(\xi)^{2}-b^{3} \alpha U^{(3)}(\xi)+2 a^{2} c \beta U^{(3)}(\xi)+6 a^{3} b U^{\prime}(\xi) U^{(3)}(\xi)+a^{4} b U^{(5)}(\xi)=0 .
$$

Integrating it once and put the integration constant equal zero, we get:

$$
-b^{3} \alpha U^{\prime \prime}(\xi)+2 a^{2} c \beta U^{\prime \prime}(\xi)+6 a^{3} b U^{\prime}(\xi) U^{\prime \prime}(\xi)+a^{4} b U^{(4)}(\xi)=0 .
$$

Balancing $U^{(4)}$ with $\left(U^{\prime}(\xi) U^{\prime \prime}(\xi)\right)$ in (20) we get $N+4=2 N+3$, then $N=1$.

\subsection{Solutions using the extended tanh function method}

From (5), the solution of (20) can be given in the form:

$$
U(\xi)=a_{0}+a_{1} \Phi(\xi)+\frac{b_{1}}{\Phi(\xi)} .
$$

Substituting (21) into (20) with (6) and collecting the coefficients of $\Phi^{i}(\xi)$, we obtain the following system:

$$
\begin{aligned}
& 24 a^{4} b a_{1}+12 a^{3} b a_{1}^{2}=0, \\
& -2 b^{3} \alpha a_{1}+4 a^{2} c \beta a_{1}+40 a^{4} b w a_{1}+24 a^{3} b w a_{1}^{2}-12 a^{3} b a_{1} b_{1}=0, \\
& -2 b^{3} \alpha w a_{1}+4 a^{2} c \beta w a_{1}+16 a^{4} b w^{2} a_{1}+12 a^{3} b w^{2} a_{1}^{2}-12 a^{3} w b a_{1} b_{1}=0, \\
& -2 b^{3} \alpha w b_{1}+4 a^{2} c \beta w b_{1}+16 a^{4} b w^{2} b_{1}+12 a^{3} b w^{2} a_{1} b_{1}-12 a^{3} b w b_{1}^{2}=0, \\
& -2 b^{3} \alpha w^{2} b_{1}+4 a^{2} c \beta w^{2} b_{1}+40 a^{4} b w^{3} b_{1}+12 a^{3} b w^{3} a_{1} b_{1}-24 a^{3} b w^{2} b_{1}^{2}=0, \\
& 24 a^{4} b w^{4} b_{1}-12 a^{3} b w^{3} b_{1}^{2}=0 .
\end{aligned}
$$

From the previous system solution, we can get the following set of solutions:

Set 1:

$$
a_{1}=-2 a, b_{1}=0, w=\frac{-b^{3} \alpha+2 a^{2} c \beta}{4 a^{4} b} .
$$

Substituting (23) in (21) with (3), we find the solution of (1):

If $w<0$, then

$$
\begin{aligned}
& u(x, y, t)=a_{0}+a \sqrt{-\frac{-b^{3} \alpha+2 a^{2} c \beta}{a^{4} b}} \operatorname{coth}\left(\frac{1}{2} \sqrt{-\frac{-b^{3} \alpha+2 a^{2} c \beta}{a^{4} b}}(a x+b y-c t)\right), \\
& u(x, y, t)=a_{0}+a \sqrt{-\frac{-b^{3} \alpha+2 a^{2} c \beta}{a^{4} b}} \tanh \left(\frac{1}{2} \sqrt{-\frac{-b^{3} \alpha+2 a^{2} c \beta}{a^{4} b}}(a x+b y-c t)\right) .
\end{aligned}
$$

If $w>0$, we get

$$
\begin{aligned}
& u(x, y, t)=a_{0}-a \sqrt{\frac{-b^{3} \alpha+2 a^{2} c \beta}{a^{4} b}} \tan \left(\frac{1}{2} \sqrt{\frac{-b^{3} \alpha+2 a^{2} c \beta}{a^{4} b}}(a x+b y-c t)\right), \\
& u(x, y, t)=a_{0}+a \sqrt{\frac{-b^{3} \alpha+2 a^{2} c \beta}{a^{4} b}} \cot \left(\frac{1}{2} \sqrt{\frac{-b^{3} \alpha+2 a^{2} c \beta}{a^{4} b}}(a x+b y-c t)\right) .
\end{aligned}
$$

Set 2:

$$
a_{1}=-2 a, b_{1}=\frac{-b^{3} \alpha+2 a^{2} c \beta}{8 a^{3} b}, w=\frac{-b^{3} \alpha+2 a^{2} c \beta}{16 a^{4} b} .
$$


If $w<0$, then

$$
\begin{aligned}
& u(x, y, t)=a_{0}+\frac{1}{2} a \sqrt{-\frac{-b^{3} \alpha+2 a^{2} c \beta}{a^{4} b}} \tanh \left(\frac{1}{4} \sqrt{-\frac{-b^{3} \alpha+2 a^{2} c \beta}{a^{4} b}}(a x+b y-c t)\right) \\
& -\frac{\left(\left(-b^{3} \alpha+2 a^{2} c \beta\right) \operatorname{coth}\left(\frac{1}{4} \sqrt{-\frac{-b^{3} \alpha+2 a^{2} c \beta}{a^{4} b}}(a x+b y-c t)\right)\right)}{2 a^{3} b \sqrt{-\frac{-b^{3} \alpha+2 a^{2} c \beta}{a^{4} b}}}, \\
& u(x, y, t)=a_{0}+\frac{1}{2} a \sqrt{-\frac{-b^{3} \alpha+2 a^{2} c \beta}{a^{4} b}} \operatorname{coth}\left(\frac{1}{4} \sqrt{-\frac{-b^{3} \alpha+2 a^{2} c \beta}{a^{4} b}}(a x+b y-c t)\right) \\
& -\frac{\left(\left(-b^{3} \alpha+2 a^{2} c \beta\right) \tanh \left(\frac{1}{4} \sqrt{-\frac{-b^{3} \alpha+2 a^{2} c \beta}{a^{4} b}}(a x+b y-c t)\right)\right)}{2 a^{3} b \sqrt{-\frac{-b^{3} \alpha+2 a^{2} c \beta}{a^{4} b}}} .
\end{aligned}
$$

If $w>0$, thus

$$
\begin{aligned}
& u(x, y, t)=a_{0}-\frac{1}{2} a \sqrt{\frac{-b^{3} \alpha+2 a^{2} c \beta}{a^{4} b}} \tan \left(\frac{1}{4} \sqrt{\frac{-b^{3} \alpha+2 a^{2} c \beta}{a^{4} b}}(a x+b y-c t)\right) \\
& +\frac{\left(\left(-b^{3} \alpha+2 a^{2} c \beta\right) \cot \left(\frac{1}{4} \sqrt{\frac{-b^{3} \alpha+2 a^{2} c \beta}{a^{4} b}}(a x+b y-c t)\right)\right)}{2 a^{3} b \sqrt{\frac{-b^{3} \alpha+2 a^{2} c \beta}{a^{4} b}}}, \\
& u(x, y, t)=a_{0}+\frac{1}{2} a \sqrt{\frac{-b^{3} \alpha+2 a^{2} c \beta}{a^{4} b}} \cot \left(\frac{1}{4} \sqrt{\frac{-b^{3} \alpha+2 a^{2} c \beta}{a^{4} b}}(a x+b y-c t)\right) \\
& -\frac{\left(\left(-b^{3} \alpha+2 a^{2} c \beta\right) \tan \left(\frac{1}{4} \sqrt{\frac{-b^{3} \alpha+2 a^{2} c \beta}{a^{4} b}}(a x+b y-c t)\right)\right)}{2 a^{3} b \sqrt{\frac{-b^{3} \alpha+2 a^{2} c \beta}{a^{4} b}}} .
\end{aligned}
$$

Set 3:

$$
a_{1}=0, b_{1}=\frac{-b^{3} \alpha+2 a^{2} c \beta}{2 a^{3} b}, w=\frac{-b^{3} \alpha+2 a^{2} c \beta}{4 a^{4} b} .
$$

If $w<0$, we obtain

$$
\begin{aligned}
& u(x, y, t)=a_{0}-\frac{\left(\left(-b^{3} \alpha+2 a^{2} c \beta\right) \tanh \left(\frac{1}{2} \sqrt{-\frac{-b^{3} \alpha+2 a^{2} c \beta}{a^{4} b}}(a x+b y-c t)\right)\right)}{a^{3} b \sqrt{-\frac{-b^{3} \alpha+2 a^{2} c \beta}{a^{4} b}}}, \\
& u(x, y, t)=a_{0}-\frac{\left(\left(-b^{3} \alpha+2 a^{2} c \beta\right) \operatorname{coth}\left(\frac{1}{2} \sqrt{-\frac{-b^{3} \alpha+2 a^{2} c \beta}{a^{4} b}}(a x+b y-c t)\right)\right)}{a^{3} b \sqrt{-\frac{-b^{3} \alpha+2 a^{2} c \beta}{a^{4} b}}} .
\end{aligned}
$$

If $w>0$, then

$$
\begin{aligned}
& u(x, y, t)=a_{0}+\frac{\left(\left(-b^{3} \alpha+2 a^{2} c \beta\right) \cot \left(\frac{1}{2} \sqrt{\frac{-b^{3} \alpha+2 a^{2} c \beta}{a^{4} b}}(a x+b y-c t)\right)\right)}{a^{3} b \sqrt{\frac{-b^{3} \alpha+2 a^{2} c \beta}{a^{4} b}}}, \\
& u(x, y, t)=a_{0}-\frac{\left(\left(-b^{3} \alpha+2 a^{2} c \beta\right) \tan \left(\frac{1}{2} \sqrt{\frac{-b^{3} \alpha+2 a^{2} c \beta}{a^{4} b}}(a x+b y-c t)\right)\right)}{a^{3} b \sqrt{\frac{-b^{3} \alpha+2 a^{2} c \beta}{a^{4} b}}} .
\end{aligned}
$$


Set 4:

$$
\begin{gathered}
a_{1}=-2 a, b_{1}=\frac{-b^{3} \alpha+2 a^{2} c \beta}{6 a^{3} b}, w=0 . \\
u(x, y, t)=a_{0}+\frac{2 a}{(a x+b y-c t)}-\frac{(a x+b y-c t)\left(-b^{3} \alpha+2 a^{2} c \beta\right)}{6 a^{3} b} .
\end{gathered}
$$

\subsection{Solutions using the simple equation method}

From (10), the solution of (20) can be given in the form:

$$
U(\xi)=A_{0}+A_{1} \phi(\xi)
$$

Substituting from (34) and the Bernoulli equation, its special cases and the Ricatti equation into (20). Collect the coefficients of $\phi^{i}(\xi)$ that have the same power and we equate them to zero.

\subsubsection{Bernoulli equation}

Substituting (34) and (11) into (20) we obtain

$$
\begin{aligned}
& -b^{3} \alpha A_{1} B_{2}^{2}+2 a^{2} c \beta A_{1} B_{2}^{2}+a^{4} b A_{1} B_{2}^{4}=0, \\
& -2 b^{3} \alpha A_{1} B_{1}^{2}+4 a^{2} c \beta A_{1} B_{1}^{2}+24 a^{3} b A_{1}^{2} B_{1} B_{2}^{2}+50 a^{4} b A_{1} B_{1}^{2} B_{2}^{2}=0, \\
& 30 a^{3} b A_{1}^{2} B_{1}^{2} B_{2}+60 a^{4} b A_{1} B_{1}^{3} B_{2}=0, \\
& -3 b^{3} \alpha A_{1} B_{1} B_{2}+6 a^{2} c \beta A_{1} B_{1} B_{2}+6 a^{3} b A_{1}^{2} B_{2}^{3}+15 a^{4} b A_{1} B_{1} B_{2}^{3}=0, \\
& 12 a^{3} b A_{1}^{2} B_{1}^{3}+24 a^{4} b A_{1} B_{1}^{4}=0 .
\end{aligned}
$$

From the previous system solution, we can get the following set of solutions:

Set 1:

$$
\begin{gathered}
A_{1}=-2 a B_{1}, B_{2}=\frac{\sqrt{b^{3} \alpha-2 a^{2} c \beta}}{a^{2} \sqrt{b}}, \\
u(x, y, t)=A_{0}+\frac{2 B_{1} \sqrt{b^{3} \alpha-2 a^{2} c \beta} \exp \left(\frac{(a x+b y-c t) \sqrt{b^{3} \alpha-2 a^{2} c \beta}}{a^{2} \sqrt{b}}\right)}{a \sqrt{b}\left(-1+B_{1} \exp \left(\frac{(a x+b y-c t) \sqrt{b^{3} \alpha-2 a^{2} c \beta}}{a^{2} \sqrt{b}}\right)\right)} .
\end{gathered}
$$

Set 2:

$$
\begin{gathered}
A_{1}=-2 a B_{1}, B_{2}=-\frac{\sqrt{b^{3} \alpha-2 a^{2} c \beta}}{a^{2} \sqrt{b}}, \\
u(x, y, t)=A_{0}-\frac{2 B_{1} \sqrt{b^{3} \alpha-2 a^{2} c \beta} \exp \left(-\frac{(a x+b y-c t) \sqrt{b^{3} \alpha-2 a^{2} c \beta}}{a^{2} \sqrt{b}}\right)}{a \sqrt{b}\left(-1+B_{1} \exp \left(-\frac{(a x+b y-c t) \sqrt{b^{3} \alpha-2 a^{2} c \beta}}{a^{2} \sqrt{b}}\right)\right)} .
\end{gathered}
$$

\section{Case one:}$$
\text { By substitution from (34) and (13) into (20) we get: }
$$$$
\begin{aligned}
& -b^{3} \alpha A_{1} B_{2}^{2}+2 a^{2} c \beta A_{1} B_{2}^{2}+a^{4} b A_{1} B_{2}^{4}=0 \\
& -2 b^{3} \alpha A_{1}+4 a^{2} c \beta A_{1}-24 a^{3} b A_{1}^{2} B_{2}^{2}+50 a^{4} b A_{1} B_{2}^{2}=0 \\
& 30 a^{3} b A_{1}^{2} B_{2}-60 a^{4} b A_{1} B_{2}=0 \\
& 3 b^{3} \alpha A_{1} B_{2}-6 a^{2} c \beta A_{1} B_{2}+6 a^{3} b A_{1}^{2} B_{2}^{3}-15 a^{4} b A_{1} B_{2}^{3}=0 \\
& -12 a^{3} b A_{1}^{2}+24 a^{4} b A_{1}=0
\end{aligned}
$$ 
From the previous system solution, we can get the following set of solutions:

Set 1:

$$
\begin{gathered}
A_{1}=2 a, B_{2}=\frac{\sqrt{b^{3} \alpha-2 a^{2} c \beta}}{a^{2} \sqrt{b}}, \\
u(x, y, t)=A_{0}+\frac{2 \sqrt{b^{3} \alpha-2 a^{2} c \beta} \exp \left(\frac{(a x+b y-c t) \sqrt{b^{3} \alpha-2 a^{2} c \beta}}{a^{2} \sqrt{b}}\right)}{a \sqrt{b}\left(-1+\exp \left(\frac{(a x+b y-c t) \sqrt{b^{3} \alpha-2 a^{2} c \beta}}{a^{2} \sqrt{b}}\right)\right)} .
\end{gathered}
$$

Set 2:

$$
\begin{gathered}
A_{1}=2 a, B_{2}=-\frac{\sqrt{b^{3} \alpha-2 a^{2} c \beta}}{a^{2} \sqrt{b}}, \\
u(x, y, t)=A_{0}-\frac{2 \sqrt{b^{3} \alpha-2 a^{2} c \beta} \exp \left(-\frac{(a x+b y-c t) \sqrt{b^{3} \alpha-2 a^{2} c \beta}}{a^{2} \sqrt{b}}\right)}{a \sqrt{b}\left(-1+\exp \left(-\frac{(a x+b y-c t) \sqrt{b^{3} \alpha-2 a^{2} c \beta}}{a^{2} \sqrt{b}}\right)\right)} .
\end{gathered}
$$

\section{Case two:}

Substituting (34) and (15) into (20) gives

$$
\begin{aligned}
& -b^{3} \alpha A_{1}+2 a^{2} c \beta A_{1}+a^{4} b A_{1}=0 \\
& -2 b^{3} \alpha A_{1}+4 a^{2} c \beta A_{1}-24 a^{3} b A_{1}^{2}+50 a^{4} b A_{1}=0 \\
& 30 a^{3} b A_{1}^{2}-60 a^{4} b A_{1}=0 \\
& 3 b^{3} \alpha A_{1}-6 a^{2} c \beta A_{1}+6 a^{3} b A_{1}^{2}-15 a^{4} b A_{1}=0 \\
& -12 a^{3} b A_{1}^{2}+24 a^{4} b A_{1}=0
\end{aligned}
$$

From the previous system solution, we can get the following set of solutions:

Set 1:

$$
\begin{gathered}
A_{1}=-2 \sqrt{\frac{-c \beta-\sqrt{b^{4} \alpha+c^{2} \beta^{2}}}{b}}, a=-\sqrt{\frac{-c \beta-\sqrt{b^{4} \alpha+c^{2} \beta^{2}}}{b}}, \\
u(x, y, t)=A_{0}-\frac{2 \sqrt{\frac{-c \beta-\sqrt{b^{4} \alpha+c^{2} \beta^{2}}}{b}} \exp \left(-\sqrt{\frac{-c \beta-\sqrt{b^{4} \alpha+c^{2} \beta^{2}}}{b}} x+b y-c t\right)}{1+\exp \left(-\sqrt{\frac{-c \beta-\sqrt{b^{4} \alpha+c^{2} \beta^{2}}}{b}} x+b y-c t\right)} .
\end{gathered}
$$

Set 2:

$$
\begin{gathered}
A_{1}=2 \sqrt{\frac{-c \beta-\sqrt{b^{4} \alpha+c^{2} \beta^{2}}}{b}}, a=\sqrt{\frac{-c \beta-\sqrt{b^{4} \alpha+c^{2} \beta^{2}}}{b}}, \\
u(x, y, t)=A_{0}+\frac{2 \sqrt{\frac{-c \beta-\sqrt{b^{4} \alpha+c^{2} \beta^{2}}}{b}} \exp \left(\sqrt{\frac{-c \beta-\sqrt{b^{4} \alpha+c^{2} \beta^{2}}}{b}} x+b y-c t\right)}{1+\exp \left(\sqrt{\frac{-c \beta-\sqrt{b^{4} \alpha+c^{2} \beta^{2}}}{b}} x+b y-c t\right)} .
\end{gathered}
$$

Set 3:

$$
\begin{gathered}
A_{1}=-2 \sqrt{\frac{-c \beta+\sqrt{b^{4} \alpha+c^{2} \beta^{2}}}{b}}, a=-\sqrt{\frac{-c \beta+\sqrt{b^{4} \alpha+c^{2} \beta^{2}}}{b}}, \\
u(x, y, t)=A_{0}-\frac{2 \sqrt{\frac{-c \beta+\sqrt{b^{4} \alpha+c^{2} \beta^{2}}}{b}} \exp \left(-\sqrt{\frac{-c \beta+\sqrt{b^{4} \alpha+c^{2} \beta^{2}}}{b}} x+b y-c t\right)}{1+\exp \left(-\sqrt{\frac{-c \beta+\sqrt{b^{4} \alpha+c^{2} \beta^{2}}}{b}} x+b y-c t\right)} .
\end{gathered}
$$


Set 4:

$$
\begin{gathered}
A_{1}=2 \sqrt{\frac{-c \beta+\sqrt{b^{4} \alpha+c^{2} \beta^{2}}}{b}}, a=\sqrt{\frac{-c \beta+\sqrt{b^{4} \alpha+c^{2} \beta^{2}}}{b}}, \\
u(x, y, t)=A_{0}+\frac{2 \sqrt{\frac{-c \beta+\sqrt{b^{4} \alpha+c^{2} \beta^{2}}}{b}}}{1+\exp \left(\sqrt{\frac{-c \beta+\sqrt{b^{4} \alpha+c^{2} \beta^{2}}}{b}} x+b y-c t\right)} .
\end{gathered}
$$

\subsubsection{Riccati equation}

By substitution from (34) and (17) into (20) we get:

$$
\begin{aligned}
& -2 b^{3} \alpha A_{1} B_{1} B_{2}+4 a^{2} c \beta A_{1} B_{1} B_{2}+12 a^{3} b A_{1}^{2} B_{1} B_{2}^{2}+16 a^{4} b A_{1} B_{1}^{2} B_{2}^{2}=0, \\
& -2 b^{3} \alpha A_{1} B_{1}^{2}+4 a^{2} c \beta A_{1} B_{1}^{2}+24 a^{3} b A_{1}^{2} B_{1}^{2} B_{2}+40 a^{4} b A_{1} B_{1}^{3} B_{2}=0, \\
& 12 a^{3} b A_{1}^{2} B_{1}^{3}+24 a^{4} b A_{1} B_{1}^{4}=0 .
\end{aligned}
$$

From the previous system solution, we can get the solution:

$$
\begin{gathered}
A_{1}=\frac{b^{3} \alpha-2 a^{2} c \beta}{2 a^{3} b B_{2}}, B_{1}=\frac{-b^{3} \alpha+2 a^{2} c \beta}{4 a^{4} b B_{2}}, \\
u(x, y, t)=A_{0}+\frac{\left(b^{3} \alpha-2 a^{2} c \beta\right) \tan \left(\frac{1}{2} \sqrt{B_{2}}(a x+b y-c t) \sqrt{\frac{-b^{3} \alpha+2 a^{2} c \beta}{a^{4} b B_{2}}}\right)}{a^{3} b \sqrt{B_{2}} \sqrt{\frac{-b^{3} \alpha+2 a^{2} c \beta}{a^{4} b B_{2}}}} .
\end{gathered}
$$

\section{Finite difference method}

In this section, we take approximations for spaces $x, y$ and time $t$ derivatives as [22, 23]:

$$
\begin{aligned}
& u_{t} \simeq \frac{c_{i, j, n+1}-c_{i, j, n}}{\Delta t} \\
& u_{x} \simeq \frac{c_{i+1, j, n}-c_{i-1, j, n}}{2 h}, \\
& u_{x x} \simeq \frac{c_{i-1, j, n}+c_{i+1, j, n}-2 c_{i, j, n}}{h^{2}}, \\
& u_{y} \simeq \frac{c_{i, j+1, n}-c_{i, j-1, n}}{2 k}, \\
& u_{y y} \simeq \frac{c_{i, j-1, n}+c_{i, j+1, n}-2 c_{i, j, n}}{k^{2}}, \\
& u_{x x t} \simeq \frac{1}{h^{2} \Delta t}\left(c_{i-1, j, n+1}-2 c_{i, j, n+1}+c_{i+1, j, n+1}-\left(c_{i-1, j, n}-2 c_{i, j, n}+c_{i+1, j, n}\right)\right), \\
& u_{x y} \simeq \frac{1}{h k}\left(c_{i+1, j+1, n}-c_{i-1, j+1, n}\right)-\left(c_{i+1, j-1, n}-c_{i-1, j-1, n}\right), \\
& u_{x x x} \simeq \frac{-c_{i-2, j, n}+2 c_{i-1, j, n}-2 c_{i+1, j, n}+c_{i+2, j, n}}{2 h^{3}}, \\
& u_{y y y} \simeq \frac{-c_{i, j-2, n}+2 c_{i, j-1, n}-2 c_{i, j+1, n}+c_{i, j+2, n}}{2 k^{3}}, \\
& u_{x x y} \simeq \frac{1}{2 h^{2} k}\left(\left(c_{i-1, j+1, n}-2 c_{i, j+1, n}+c_{i+1, j+1, n}\right)-\left(c_{i-1, j-1, n}-2 c_{i, j-1, n}+c_{i+1, j-1, n}\right)\right) \\
& \vdots
\end{aligned}
$$


Table 1: Comparison between the numerical results with the analytical solution

\begin{tabular}{l|l|l|l}
\hline$x$ & Numerical solution & Analytical solution & Absolute error \\
\hline-10.0 & 0.899689 & 0.899689 & 0.00000 \\
\hline-8.0 & 0.902401 & 0.901921 & $4.79189 \mathrm{E}-4$ \\
\hline-6.0 & 0.902788 & 0.902296 & $4.92636 \mathrm{E}-4$ \\
\hline-4.0 & 0.902817 & 0.902359 & $4.58368 \mathrm{E}-4$ \\
\hline-2.0 & 0.902779 & 0.902369 & $4.09901 \mathrm{E}-4$ \\
\hline 0.0 & 0.902724 & 0.902371 & $3.53541 \mathrm{E}-4$ \\
\hline 2.0 & 0.902662 & 0.902371 & $2.91129 \mathrm{E}-4$ \\
\hline 4.0 & 0.902595 & 0.902371 & $2.23846 \mathrm{E}-4$ \\
\hline 6.0 & 0.902524 & 0.902371 & $1.52841 \mathrm{E}-4$ \\
\hline 8.0 & 0.902451 & 0.902371 & $7.93110 \mathrm{E}-5$ \\
\hline 10.0 & 0.902371 & 0.902371 & 0.00000
\end{tabular}

Table 2: Comparison between the numerical results with the analytical solution

\begin{tabular}{l|l|l|l}
\hline$x$ & Numerical solution & Analytical solution & Absolute error \\
\hline-10.0 & 0.096130 & 0.096130 & 0.00000 \\
\hline-8.0 & 0.104001 & 0.103995 & $5.00902 \mathrm{E}-6$ \\
\hline-6.0 & 0.118948 & 0.118939 & $1.00629 \mathrm{E}-5$ \\
\hline-4.0 & 0.136836 & 0.136823 & $1.41558 \mathrm{E}-5$ \\
\hline-2.0 & 0.146707 & 0.146711 & $1.23657 \mathrm{E}-5$ \\
\hline 0.0 & 0.142060 & 0.142070 & $1.01504 \mathrm{E}-5$ \\
\hline 2.0 & 0.129208 & 0.129211 & $5.42566 \mathrm{E}-6$ \\
\hline 4.0 & 0.115798 & 0.115799 & $2.59471 \mathrm{E}-6$ \\
\hline 6.0 & 0.105002 & 0.105003 & $1.72930 \mathrm{E}-6$ \\
\hline 8.0 & 0.097735 & 0.097736 & $1.70834 \mathrm{E}-6$ \\
\hline 10.0 & 0.094660 & 0.094660 & 0.00000
\end{tabular}

Now, we assume that $u$ the exact solution at the grid point $\left(x_{i}, y_{j}, t_{n}\right)$ and $c_{i, j, n}$ is the numerical solution at the same point. Substituting (57) into (1) we get the system of difference equations. We can solve this system to find the numerical values of $c_{i, j, n}$.

\subsection{The numerical results}

Now, we will intreduce some numerical resultes for the (2+1)-dimensional Date-Jimbo-Kashiwara-Miwa equation. In Table 1 we introduce comparison between the numerical results with the analytical solution (30) at $\alpha=0.1, \beta=0.1$, $a=0.7, b=2, c=0.2, a_{0}=0.01, \theta=0.1, y=6.0$. In Fig. 10 we introduce analytical and numerical solutions for (1) at $\alpha=0.1, \beta=0.1, \Delta t=0.001, h=1, k=1, t=0.01 a=0.7, b=2, c=0.2, a_{0}=0.01, \theta=0.1, y=1.0$.

In Table 2 we introduce comparison between the numerical results with the analytical solution (30) at $\alpha=0.01, \beta=0.1$, $a=0.7, b=0.2, c=0.2, A_{0}=0.01, B_{1}=0.2, \theta=0.3, y=6.0$. In Fig. 11 we introduce analytical and numerical solutions for (1) at $\alpha=0.01, \beta=0.1, a=0.7, b=0.2, c=0.2, A_{0}=0.01, B_{1}=0.2, \theta=0.3, h=k=1, y=6.0, \Delta t=$ $0.001, t=0.01$.

In Table 3 we introduce comparison between the numerical results with the analytical solution (53) at $\alpha=0.1, \beta=$ $0.1, b=2, c=0.2, A_{0}=0.01, \theta=0.1$. In Fig. 12 we introduce analytical and numerical solutions for (1) at $\alpha=0.1, \beta=$ $0.1, b=2, c=0.2, A_{0}=0.01, \theta=0.1 t=0.01, \Delta t=0.001, h=k=1.0, y=6.0$. 
Table 3: Comparison between the numerical results with the analytical solution

\begin{tabular}{l|l|l|l}
\hline$x$ & Numerical solution & Analytical solution & Absolute error \\
\hline-10.0 & 1.56488 & 1.56488 & 0.00000 \\
\hline-8.0 & 1.58419 & 1.58319 & $1.00568 \mathrm{E}-3$ \\
\hline-6.0 & 1.58812 & 1.58702 & $1.09763 \mathrm{E}-3$ \\
\hline-4.0 & 1.58886 & 1.58781 & $1.05164 \mathrm{E}-3$ \\
\hline-2.0 & 1.58893 & 1.58797 & $9.57979 \mathrm{E}-4$ \\
\hline 0.0 & 1.58884 & 1.58801 & $8.36084 \mathrm{E}-4$ \\
\hline 2.0 & 1.58871 & 1.58802 & $6.93327 \mathrm{E}-4$ \\
\hline 4.0 & 1.58855 & 1.58802 & $5.34539 \mathrm{E}-4$ \\
\hline 6.0 & 1.58838 & 1.58802 & $3.63934 \mathrm{E}-4$ \\
\hline 8.0 & 1.58820 & 1.58802 & $1.85530 \mathrm{E}-4$ \\
\hline 10.0 & 1.58802 & 1.58802 & 0.00000 \\
\hline
\end{tabular}

\section{Graphical illustrations}

Herein,we present some figures in the two-dimensional, three-dimensional and countours to illustrate the solutions that we have got. Some of the analytical solutions are presented in Figs. 1, 2, 3, 4, 5, 6, 7, 8 and 9, while the accuracy of the methods was compared to the solution given in [1-3] is shown in Figs. 1, 2, 3, 4, 5, 6, 7, 8 and 9, respectively. In Fig. 1, we introduce the graph of (24) using the extended tanh method at $\alpha=0.1, \beta=0.1, a=0.2, b=-0.6, c=0.1, a_{0}=$ $0.001, \theta=0.1$. Graph of (30) using the extended tanh method at $\alpha=0.1, \beta=0.1, a=0.7, b=2, c=0.2, a_{0}=$ $0.01, \theta=0.1$ was presented in Fig. 2, we introduce the graph of (37) using the simple equation method at $\alpha=0.1, \beta=$ $0.1, a=0.5, b=0.2, c=0.1, A_{0}=0.01, B_{1}=0.2, \theta=0.1 \mathrm{In}$ Fig. 3, we present the graph of (39) using the simple equation method at $\alpha=0.01, \beta=0.1, a=0.7, b=0.2, c=0.2, A_{0}=0.01, B_{1}=0.2, \theta=0.3$ in Fig. 4. Graph of (47) using the simple equation method at $\alpha=0.1, \beta=0.2, b=-0.7, c=0.1, A_{0}=0.01, \theta=0.1$ is presented in Fig. 5, we presente the Graph of (49) using the simple equation method at $\alpha=0.1, \beta=0.1, b=-0.1, c=0.1, A_{0}=0.1, \theta=0.1$ in Fig. 6. Graph of (51) using the simple equation method at $\alpha=0.1, \beta=0.1, b=2, c=0.2, A_{0}=0.1, \theta=0.1$ is presented in Fig. 7 and the graph of (53) using the simple equation method at $\alpha=0.1, \beta=0.1, b=2, c=0.2, A_{0}=0.1, \theta=0.1$ was introduced in Fig. 8. Finally, we give the graph of (56) using the simple equation method at $\alpha=0.1, \beta=0.1$, $b=-0.1, a=0.4, c=0.1, A_{0}=0.01, B_{2}=0.02, \theta=0.1$ in Fig. 9. In addition, some graphs for numerical solutions were introduced in Figs. 10, 11 and 12.
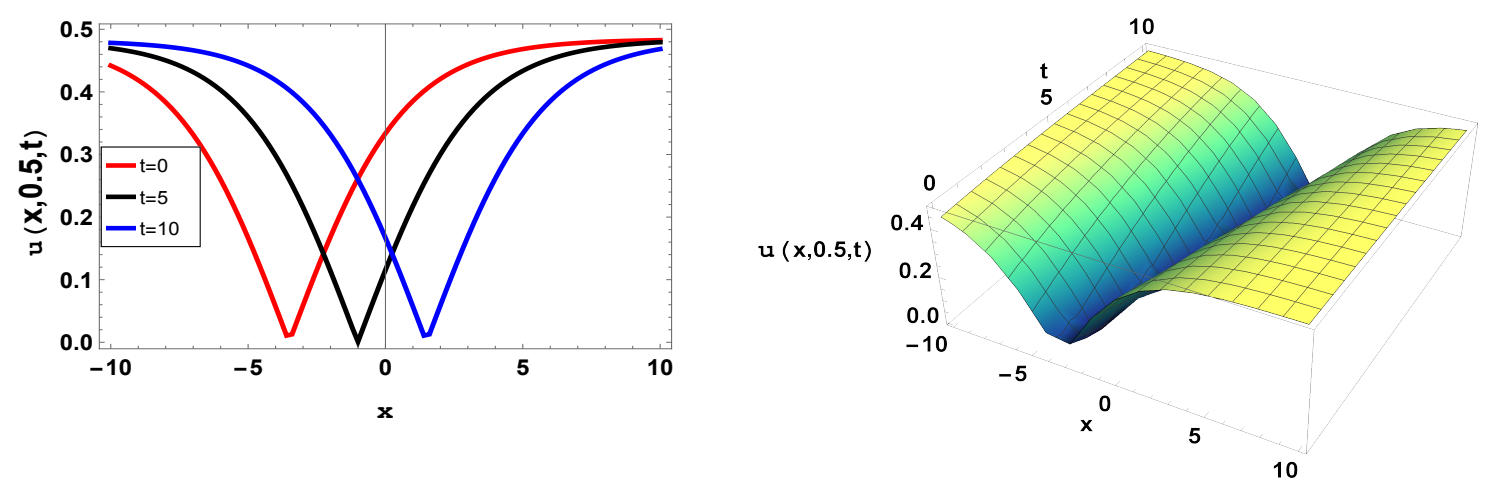

Figure 1: Graph of (24) using the extended tanh method at $\alpha=0.1, \beta=0.1, a=0.2, b=-0.6, c=0.1, a_{0}=0.001, \theta=0.1$. 

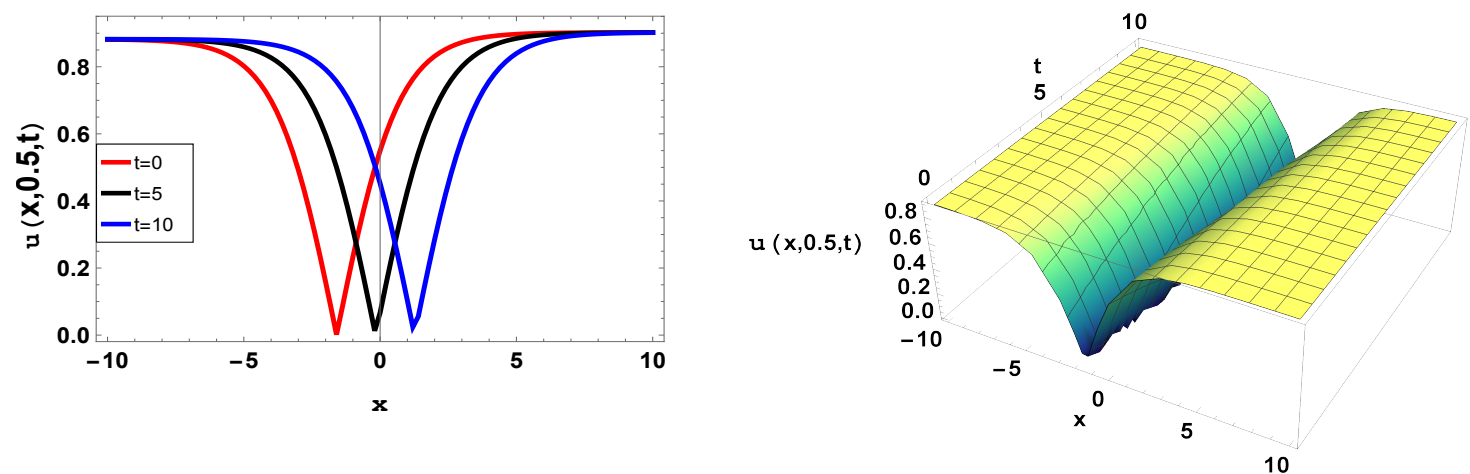

Figure 2: Graph of (30) using the extended tanh method at $\alpha=0.1, \beta=0.1, a=0.7, b=2, c=0.2, a_{0}=0.01, \theta=0.1$.
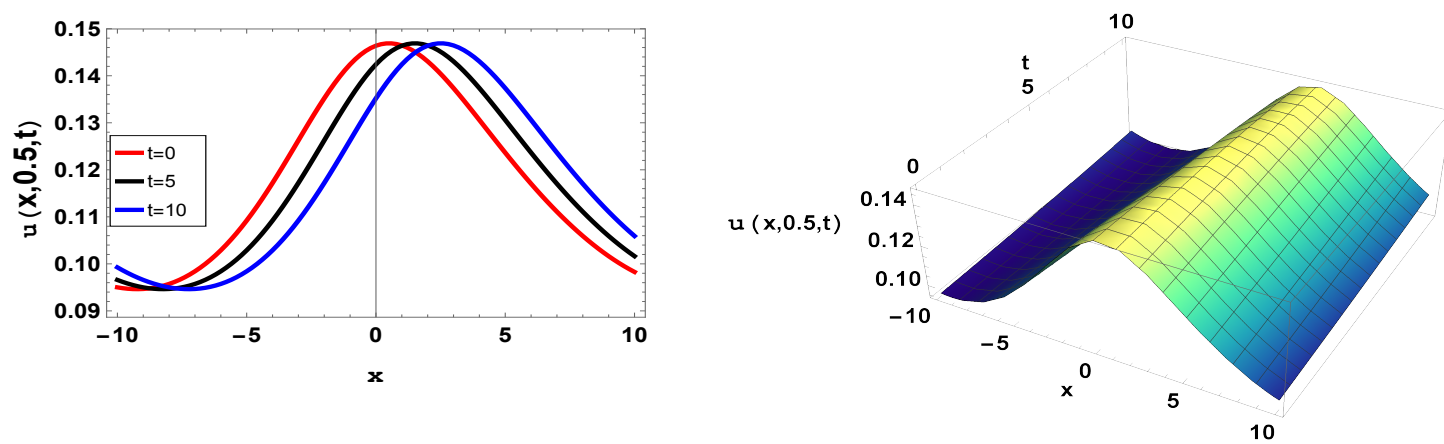

Figure 3: Graph of (37) using the simple equation method at $\alpha=0.1, \beta=0.1, a=0.5, b=0.2, c=0.1, A_{0}=0.01, B_{1}=0.2, \theta=0.1$.
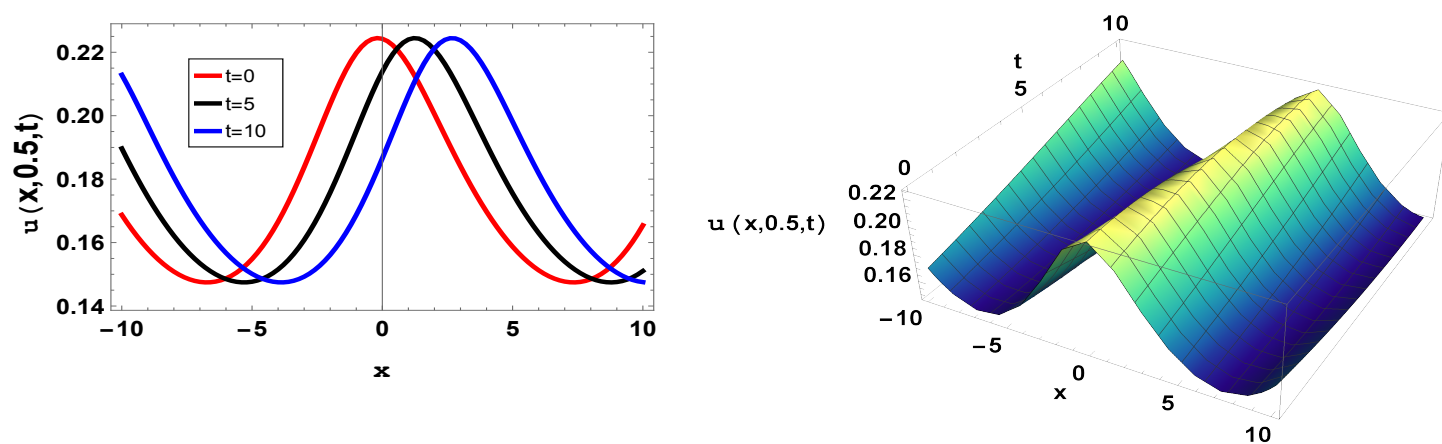

Figure 4: Graph of (39) using the simple equation method at $\alpha=0.01, \beta=0.1, a=0.7, b=0.2, c=0.2, A_{0}=0.01, B_{1}=0.2, \theta=0.3$. 

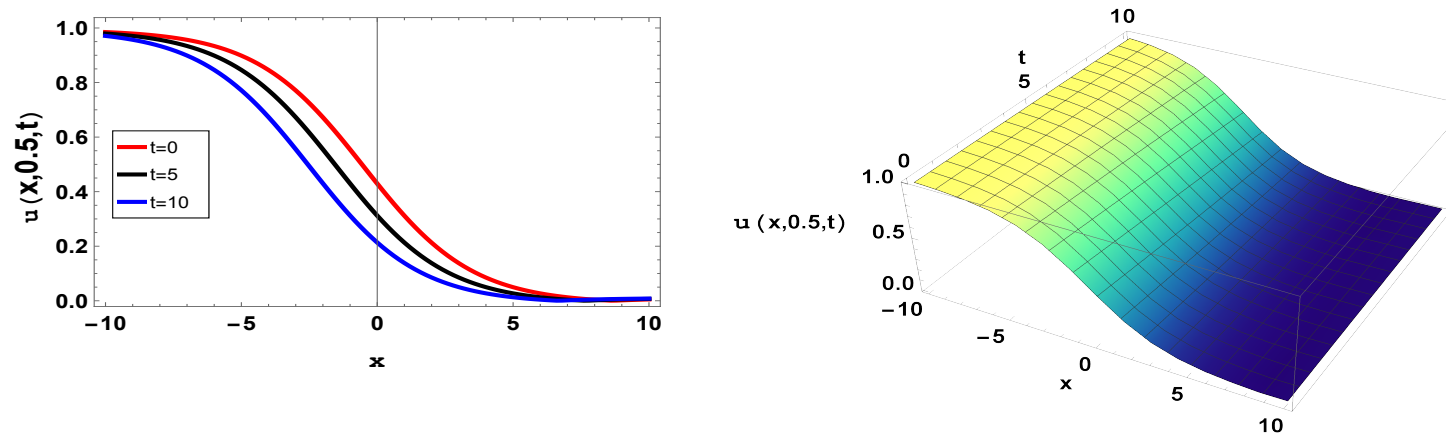

Figure 5: Graph of (47) using the simple equation method at $\alpha=0.1, \beta=0.2, b=-0.7, c=0.1, A_{0}=0.01, \theta=0.1$.
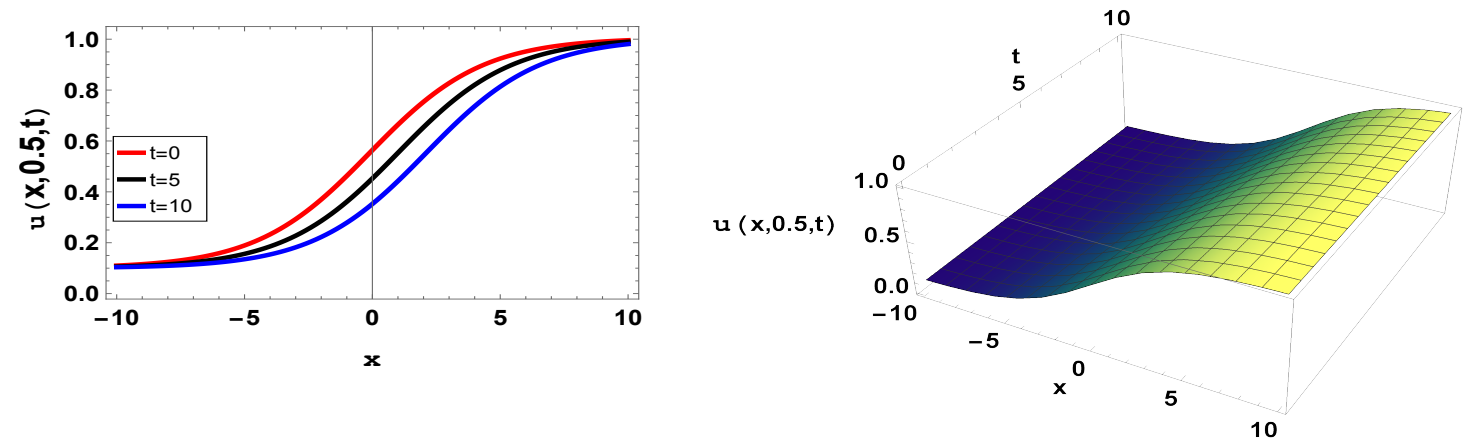

Figure 6: Graph of (49) using the simple equation method at $\alpha=0.1, \beta=0.1, b=-0.1, c=0.1, A_{0}=0.1, \theta=0.1$.
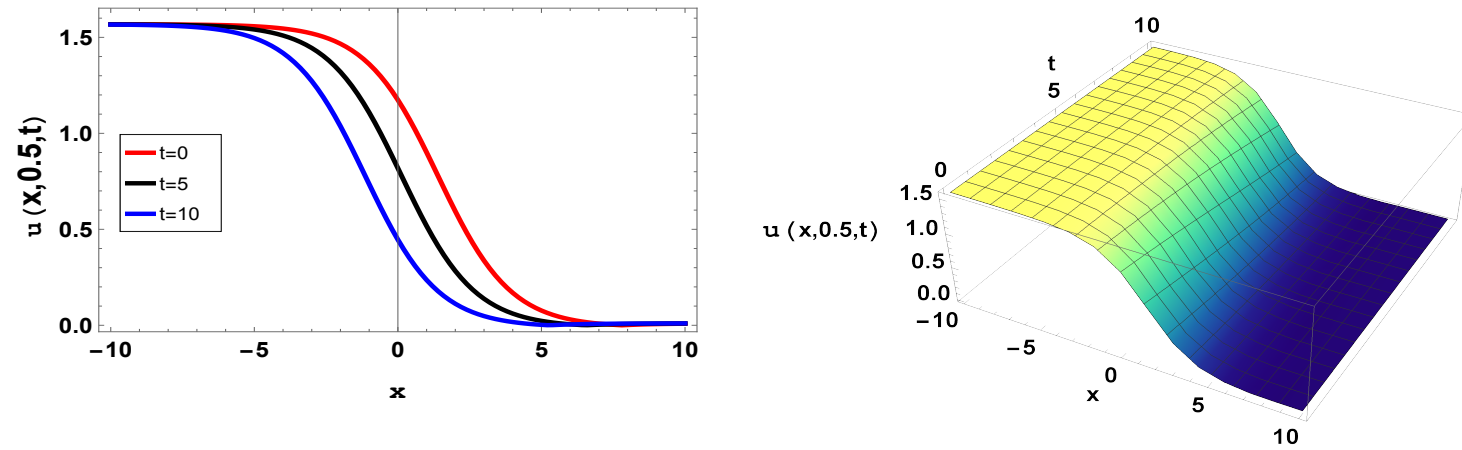

Figure 7: Graph of (51) using the simple equation method at $\alpha=0.1, \beta=0.1, b=2, c=0.2, A_{0}=0.01, \theta=0.1$. 

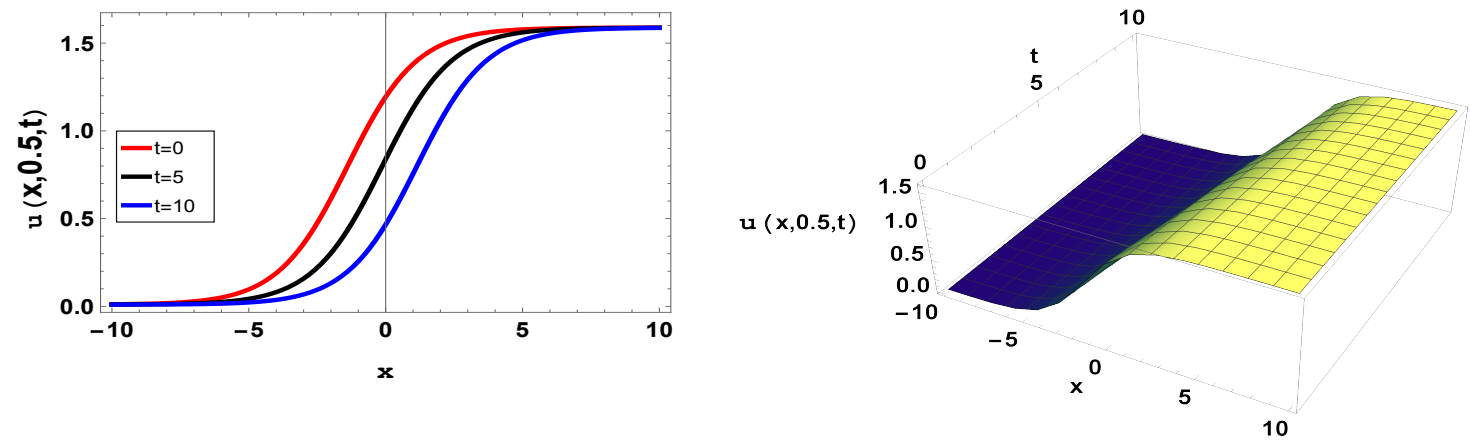

Figure 8: Graph of (53) using the simple equation method at $\alpha=0.1, \beta=0.1, b=2, c=0.2, A_{0}=0.01, \theta=0.1$.
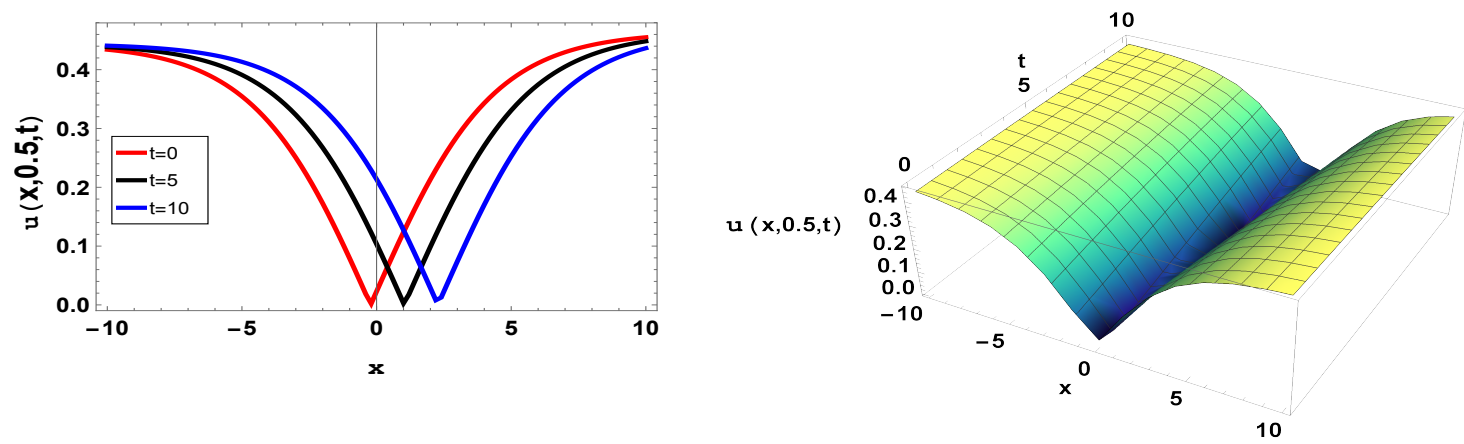

Figure 9: Graph of (56) using the simple equation method at $\alpha=0.1, \beta=0.1, b=-0.1, a=0.4, c=0.1, A_{0}=0.01, B_{2}=0.02, \theta=0.1$. 


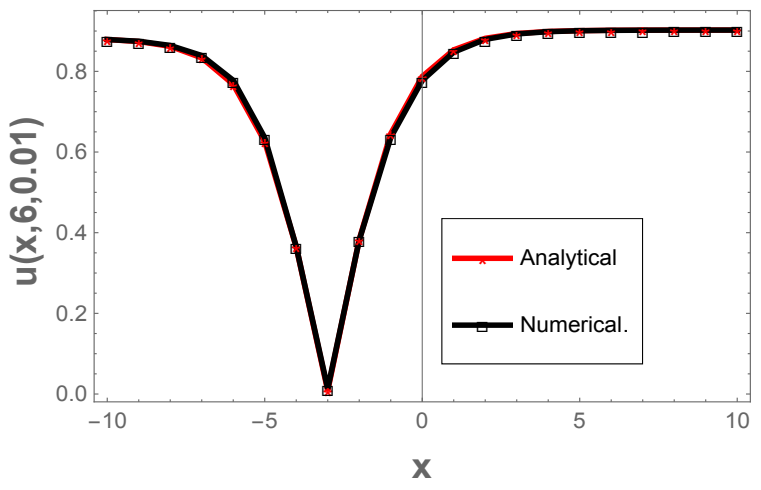

Figure 10: Graph of analytical and numerical solutions for (1) at $\alpha=0.1, \beta=0.1, \Delta t=0.001, h=1, k=1 a=0.7, b=2, c=$ $0.2, a_{0}=0.01, \theta=0.1$.

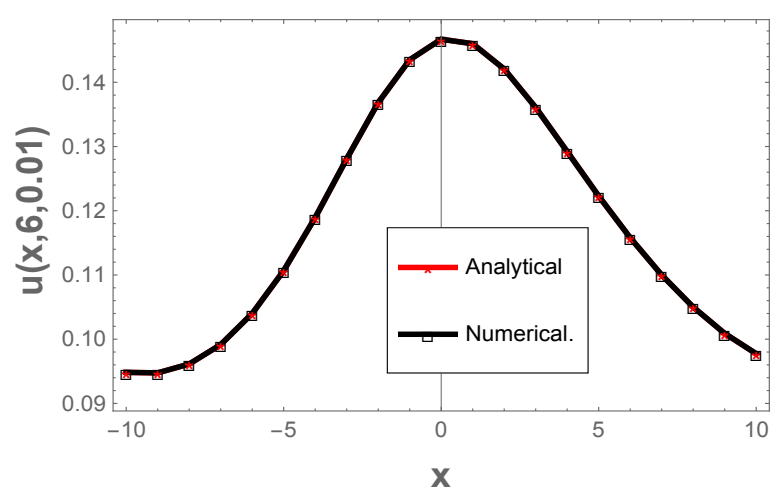

Figure 11: Graph of analytical and numerical solutions for (1) at $\alpha=$ $0.01, \beta=0.1, a=0.7, b=0.2, c=0.2, A_{0}=0.01, B_{1}=0.2, \theta=$ $0.3, h=k=1, \Delta t=0.001$.

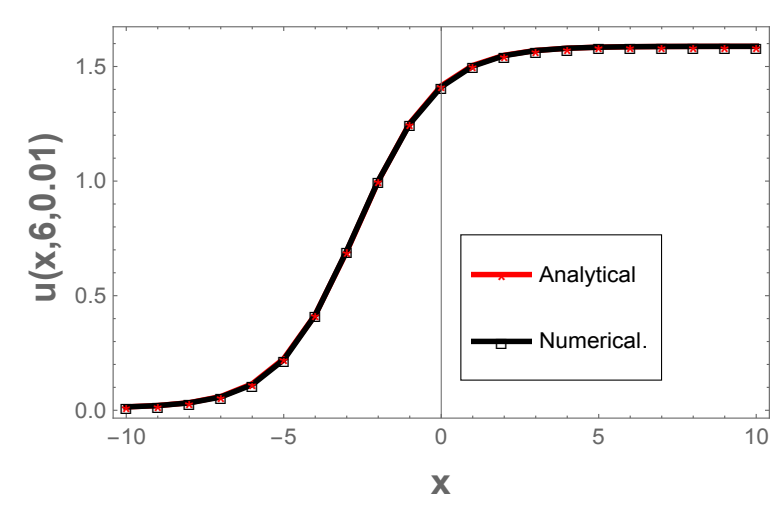

Figure 12: Graph of analytical and numerical solutions for (1) at $\alpha=0.1, \beta=0.1, b=2, c=0.2, A_{0}=0.01, \theta=0.1$ $\Delta t=0.001, h=k=1.0$.

\section{Conclusion}

In this work, the $(2+1)$-dimensional Date-JimboKashiwara-Miwa (DJKM) equation, is studied. The analytical soliton solutions of the DJKM equation were derived using the extended tanh function method and the simple equation method. The numerical treatment of that equation was conducted by the finite difference method. The accuracy of our results was illustrated by showing proper tables and figures that show the power of the techniques that were used. The work adds more analytical and numerical results in addition to the other existing results that can be found in the literature.

\section{References}

[1] Hajar FI, Hasan B, Choonkil P, Osman MS. M-lump, N-soliton solutions, and the collision phenomena for the $(2+1)$ - dimensional Date-Jimbo-Kashiwara-Miwa equation. Results Phys. 2020;19:103329.

[2] Wazwaz AM. A (2+1)-dimensional time-dependent DateJimbo-Kashiwara-Miwa equation: painlevé integrability and multiple soliton solutions. Comput Math Appl. 2020;79(4):1145-9.

[3] Berat K. Application of the improved $\tan (\varphi(\xi) / 2)$-expansion method for solving date-Jimbo-Kashiwara-Miwa equation. New Trends in Mathematical Sciences. 2019;7(1):90-7.

[4] Adem AR, Yildirim Y, Yasar E. Complexiton solutions and soliton solutions: (2+1)-dimensional Date-Jimbo-Kashiwara-Miwa equation. Pramana. 2019;92(3):1-12.

[5] Sajid N, Akram G. The Application of the $\exp (-\phi(\xi))$ Expansion Method for Finding the Exact Solutions of Two Integrable Equations, Hindawi. Math Probl Eng. 2018;12:1-10.

[6] Abdullahi RA, Yakup Y, Emrullah Y. Complexiton solu- tions and soliton solutions:(2+1)-dimensional-Date-Jimbo- KashiwaraMiwa equation. Pramana. 2019;92(3):36.

[7] Yuan YQ, Tian B, Sun WR, Chai J, Liu L. Wronskian and Grammian solutions for a (2+1)-dimensional Date-Jimbo- KashiwaraMiwa equation. Comp Math Appl Int J. 2017;74(4):873-9.

[8] Li J, Tiecheng X. A Riemann-Hilbert approach to the Kundunonlinear Schrödinger equation and its multi-component generalization. J Math Anal Appl. 2021;500(2):125109.

[9] Zhou-Zheng K., Tie-Cheng X., Construction of abundant solutions of the (2+1)-dimensional time-dependent Date-JimboKashiwara-Miwa equation, 2020, 103: 106163.

[10] Raslan KR, Ali KK. andKhalidK.Ali,Anewstructureformulations for cubic B-spline collocation method in three and four- dimensions. Nonlinear Eng. 2020;9(1):432-48.

[11] Asim Z, Hadi R, Khalid K. Ali, On finite series solutions of conformable time-fractional Cahn-Allen equation. Nonlinear Eng. 2020;9(1):194-200.

[12] Aly RS, Khalid K. Ali, Jian-Guo L., New optical soliton solutions for Fokas-Lenells dynamical equation via two various methods. Mod Phys Lett B. 2021:2150196. 
[13] Seydi BG, Liquan M, Khalid K. Ali, Two efficient methods for solving the generalizedregularized long wave equa- tion. Appl Anal. 2021:1-22.

[14] Asit S, Khalid K. Ali, Hadi R., Yogen G., Analytical optical pulses and bifurcation analysis for the traveling optical pulses of the hyperbolic nonlinear Schrödinger equation. Opt Quantum Electron. 2021;53(3):150.

[15] Khalid K. Ali, Mohamed S. O., Haci M. B., Nasser S. El., Esin I., Analytical and numerical study of the HIV-1 infection of CD4+

T-cells conformable fractional mathematical model that causes acquired immunodeficiency syndrome with the effect of antiviral drug therapy. Math Methods Appl Sci. 2020:1-17.

[16] Choonkil P, Nuruddeen RI, Khalid K. Ali, Lawal M., Osman M.S. and Dumitru B., Novel hyperbolic and exponential ansatz methods to the fractional fifth-order Korteweg'de Vries equations, Adv Diff Equat. 2020;2020:627.

[17] Wazwaz A. M., The extended tanh method for abundant solitary wave solutions of nonlinear wave equations. Appl Math Comput. 2007;187:1131-42.
[18] Raslan KR, Khalid K. AliandMuhannadA.S.,Solvingthe SpaceTime Fractional RLW and MRLW Equations Using Modi- fied Extended Tanh Method with the Riccati Equation. Brit J Math Comput Sci. 2017;21(4):1-15.

[19] Raslan KR, Khalid K. Ali and Muhannad A. S., The modified extended tanh method with the Riccati equation for solving the space-time fractional EW and MEW equations. Chaos Solitons Fractals. 2017;103:404-9.

[20] Taher AN. Simple equation method for nonlinear partial differential equations and its applications. J Egypt Math Soc. 2016;24(2):204-9.

[21] Yun-Mei Z., Ying-Hui H., and Yao L., The Simplest Equation Method and Its Application for Solving the Nonlinear NLSE, KGZ, GDS, DS, and GZ Equations. J Appl Math. 2013;960798:1-7.

[22] Raslan KR, Khalid K. Ali, Numerical study of MHD-ductflow using the two-dimensional finite difference method. Appl Math Inf Sci. 2020;14:1-5.

[23] EL-Danaf TS, Raslan KR, Khalid K Ali. New Numerical treatment for the Generalized Regularized Long Wave Equation based on finite difference scheme. Int J S Comp Eng. 2014;4:16-24. 